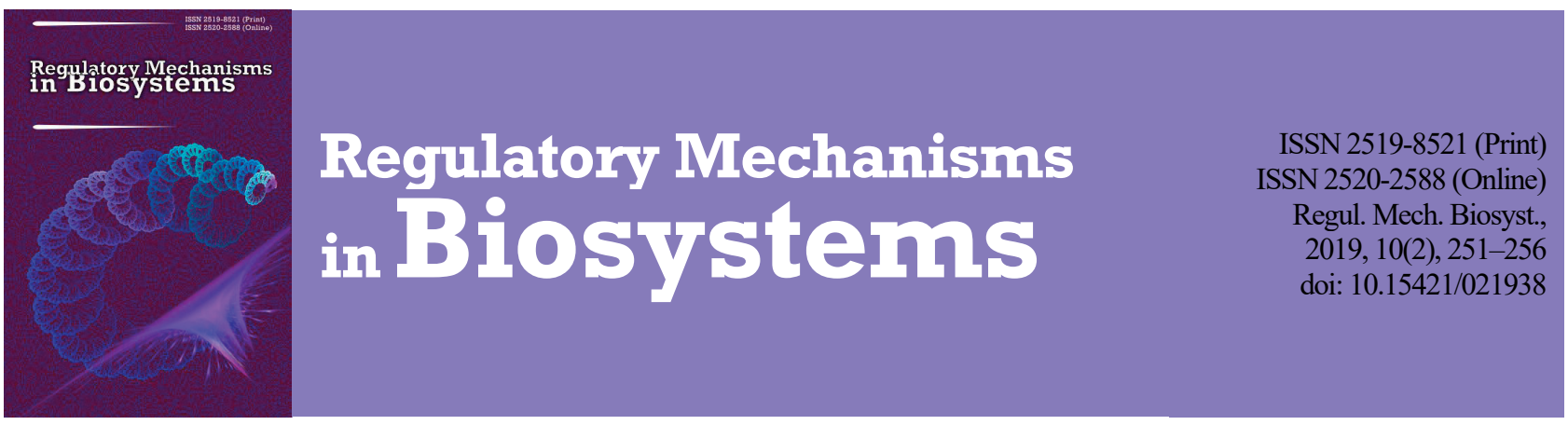

\title{
Influence of cell-free extracts of Bifidobacterium bifidum and Lactobacillus reuteri on proliferation and biofilm formation by Escherichia coli and Pseudomonas aeruginosa
}

\author{
O. V. Knysh*, O. Y. Isayenko*, Y. V. Voyda**, O. O. Kizimenko***, Y. M. Babych* \\ *I. I. Mechnikov Institute of Microbiology and Immunology of National Academy of Medical Sciences of Ukraine, Kharkiv, Ukraine \\ **Kharkiv Medical Academy of Postgraduate Education, Kharkiv, Ukraine \\ ***Ukrainian Medical Stomatological Academy, Poltava, Ukraine
}

Article info

Received 12.03.2019

Received in revised form 14.04.2019

Accepted 17.04.2019

I. I. Mechnikov Institute

of Microbiology and Immunology

of National Academy of Medical

Sciences of Ukraine.

Pushkins ka st., 14/16,

Kharkiv, 61057, Ukraine.

Tel.: +38-068-210-47-10.

E-mail:knysh_oksana@ukr.net

Kharkiv Medical Academy of Postgraduate Education, Amosova st., 58 ,

Kharkiv, 61176, Ukraine.

Ukrainian Medical Stomatological Academy, Shevchenko st., 23,

Poltava, 36011, Ukraine.

Knysh, O. V., Isayenko, O. Y., Voyda, Y. V., Kizimenko, O. O., \& Babych, Y. M. (2019). Influence of cell-free extracts of Bifidobacterium bifidum and Lactobacillus reuteri on proliferation and biofilm formation by Escherichia coli and Pseudomonas aeruginosa. Regulatory Mechanisms in Biosystems, 10(2), 251-256. doi:10.15421/021938

The development of new effective preparations for the correction of microecological disorders based on probiotic derivatives requires a comprehensive study of the biological activity of the latter. We studied the proliferative activity and biofilm formation by clinical isolates: Escherichia coli and Pseudomonas aeruginosa under the influence of cell-free extracts containing structural components and metabolites of the Bifidobacterium bifidum and Lactobacillus reuteri probiotic strains. Cell-free extracts were obtained from disintegrates and cultures of probiotics. Disintegrates were prepared by cyclic freezing-thawing of probiotic cell suspensions. The cultures were obtained by cultivating probiotic microorganisms in their own disintegrates. The obtained disintegrates and cultures were filtered. The proliferative activity of the test cultures was studied using the spectrophotometric microtiter plate method after an hour-long exposure in undiluted cell-free extracts and subsequent cultivation in a nutrient medium containing $30 \% \mathrm{vol}$ of the studied extracts at $37{ }^{\circ} \mathrm{C}$ for 24 hours. The biofilm formation of the test cultures was studied with $30 \%$ vol content of cell-free extracts in the cultivation medium using the spectrophotometric microtiter plate method. All the studied extracts exerted a similar effect on the proliferative activity and biofilm formation by $E$. coli and $P$. aeruginosa. Exposure of the test cultures in all undiluted extracts during an hour led to a significant decrease in the optical density of the test samples: optical density of the test wells ranged from $36.5 \%$ to $49.8 \%$ of the control wells. The test cultures that were exposed to the extracts: filtrate of $L$. reuteri disintegrate (L), filtrate of B. bifidum disintegrate (B) and filtrate of B. bifidum culture, grown in B. bifidum disintegrate (MB) after dilution and subsequent cultivation over the next 24 hours completely restored the ability to proliferate. The proliferative activity of the test cultures that were exposed to the extracts: filtrate of $L$. reuteri culture, grown in $L$. reuteri disintegrate $(\mathrm{ML})$ and filtrate of $L$. renteri culture, grown in $L$. reuteri disintegrate supplemented with $0.8 \mathrm{M}$ glycerol and $0.4 \mathrm{M}$ glucose (MLG), was significantly inhibited after dilution and subsequent cultivation. The inhibition indices calculated for the ML extract were: $25.9 \%$ (E. coli) and $53.0 \%$ (P. aeruginosa). Inhibition indices calculated for the MLG extract were: $62.0 \%(E$. coli $)$ and $96.9 \%(P$. aeruginos $a)$. MLG extract had more pronounced inhibitory effect on the proliferation of the test cultures than ML extract. All the studied extracts exerted significant inhibitory effect on the biofilm formation of the test cultures. Analysis of the results of the study shows that cell-free extracts of $L$. reuteri culture grown in its disintegrate without supplementation or supplemented with glycerol and glucose have the highest antimicrobial activity and can be used as metabiotics to prevent overgrowth of potentially pathogenic bacteria, as well as inoculation and proliferation of pathogenic gram-negative bacteria in the gastrointestinal tract. They can be used alone or in combination with cellular probiotics to enhance their probiotic action. This study encourages further careful investigation of the biochemical composition of cell-free extracts and clarifying the mechanism of their action.

Keywords: probiotic derivatives; lactic acid bacteria; gram-negative bacteria; metabiotics.

\section{Introduction}

Gut microbiota plays a key role in maintaining human health. Normal gut microflora provides colonization resistance and is involved in the regulation of digestion, immune, metabolic processes and neurobehavioral traits. "Health-promoting" microbes control the overgrowth of potentially pathogenic bacteria and prevent the diseases they cause (Harmsen \& de Goffau, 2016; Khodaii et al., 2017; Valdes et al., 2018). Balanced microbial composition is characterized by predominance of obligate anaerobic bacteria belonging to the classes Bacteroidia (phylum Bacteroidetes) and Clostridia (phylum Firmicutes). Minor constituents include members of the phyla Actinobacteria, Proteobacteria, Fusobacteria and members of other phylogenetic groups. The imbalance between members of the intestinal microbe community and host organism leads to dysbiosis. Microbiota disturbance can be caused by various factors: diet, toxins, drugs and pathogens. Pathogens have the greatest potential among these factors (Carding et al., 2015). Microbial imbalance usually is characterized by increased abundance of Proteobacteria belonging to the families Enterobacteriaceae, Pasteurellaceae, and Pseudomonadaceae. Dysbiosis accompanies inflammatory bowel diseases of various etiologies and may develop as a result of broad-spectrum antimicrobial chemotherapy (Winter \& Bäumler, 2014; Stecher, 2015; Litvak et al., 2017). Inflammation of the intestinal mucosa is accompanied by dysfunction of the gut barrier, manifested by the hyperpermeability of the mucosa epithelium. Membrane lipopolysacharides of the gram-negative bacteria penetrate through the intestinal mucosa into the 
blood stream, induce metabolic endotoxaemia, inflammation, impaired glucose metabolism and insulin resistance, obesity, and contribute to the development of metabolic syndrome, type II diabetes, inflammatory bowel diseases, autoimmune pathology and carcinogenesis. Dysbiosis aggravates nosocomial infections, sepsis, and multiple organ dysfunction syndrome (Halmos \& Suba, 2016; Wischmeyer et al., 2016; Yu, 2018).

The species Escherichia coli belongs to the Enterobacteriaceae family. It is predominant among facultative anaerobic bacteria of the gastrointestinal tract. This species includes of diverse strains of bacteria. Most of the strains are harmless and are an important part of a healthy gut microbiota. They have beneficial effects on some gut disorders. On the one hand, E. coli plays an important role in maintaining homeostasis between host and its associated microbiome, on the other hand, this microorganism is involved in development of dysbiosis. E. coli mostly lives in environment of multispecies biofilm and represents less than $1 \%$ of the human intestinal microbiome. However, in various cases of gut inflammation an overgrowth of $E$. coli and its significant abundance in the fecal microbiota (up to $80-90 \%$ ) can be observed. In environment of chronic intestinal inflammation, accompanied by increased fecal fat and the generation of host-derived nitrates, clonal proliferation of $E$. coli occurs. Such clonal expansion is detrimental to the balance between the microbiota and the host. This overgrowth of $E$. coli contributes to the inflammatory process and requires new therapeutic approaches that can prevent the proliferation of potentially harmful microorganisms and improve patient outcomes (Winter \& Bäumler, 2014; Martinez de la Peña et al., 2016; Matamouros et al., 2018). Some E. coli strains are pathogenic. Pathogenic E. coli strains are categorized into pathotypes. Six pathotypes belong to diarrheagenic $E$. coli. They can cause not only diarrhea, but also diseases outside the intestinal tract (urinary tract, nervous and respiratory system diseases). "Beneficial" gut flora can interfere with adhesion, invasion and overgrowth of $E$. coli, preventing the initiation and inhibiting the development of an infectious process (Gomes et al., 2016; Khodaii et al., 2017).

Pseudomonas aeruginosa belongs to the Pseudomonadaceae family. This opportunistic pathogen causes serious infections in hospital patients and immunocompromised people. $P$. aeruginosa also is considered as gut-associated bacterium. The fact that this species the most frequently identified in the feces of patients with irritable bowel syndrome indicates its involvement in the pathogenesis of this disease and its relation to intestinal dysbiosis (de Freitas et al., 2018). P. aeruginosa has the same ability to exploit inflammatory by products for its own benefit as $E$. coli. It also can utilize host-derived nitrates for anaerobic respiration, growth and bloom (Scales et al., 2016). P. aeruginosa produces quorum sensing (QS) signals that modulate the expression of virulence factors, enhance the biofilm maturation and actively inhibit innate immune responses in vitro and in vivo. QS signals which down regulate the production of tumor necrosis factor (TNF- $\alpha$ ) in stimulated macrophages, contributes to chronization of infection process (Lin \& Zhang, 2017). The severity of most infections in humans caused by $P$. aeruginosa is correlated with their ability to form biofilms (Sharma et al., 2018).

There is a huge arsenal of microbiota manipulation tools. For the recovery of the normal composition of the gut microflora in at-risk people and patients at risk people and patients are assigned to diet, probiotics, prebiotics, symbiotics, fecal microbiotatransplantation and phage therapy (Carding et al., 2015; Fuentes \& deVos, 2016; McCarville et al., 2016). The lack of effectiveness of the existing methods for the correction of microecological disturbances makes it necessary to continue the search for more effective preparations. In recent years, high hopes have been pinned on derivatives of probiotics: structural components and metabolites of probiotic cells. They can significantly affect the microbiome, host physiology, metabolism and signaling pathways. They have been developed as "metabiotics", "biological drugs", "biogenics", "cell free supernatants" (CFS), "postbiotics", "heat-killed probiotics" or "pharmacobiotics" (Shenderov, 2013; Bitto \& KaparakisLiaskos, 2017; Singh et al., 2018). The development of therapeutic agents based on bacterial derivatives for the correction of microecological disorders and the correct approaches to their use in clinical practice should be based on understanding of the mechanism of their action. In our previous study we have shown that cell-free extracts containing structural components and metabolites of probiotic B. bifidum and L. reuteri strains have a significant bifidogenic effect (Knysh, 2019). The present work was focused on studying the effect of cell-free extracts of probiotic origin on the basic physiological functions of opportunistic gram-negative microorganisms involved in the pathogenesis of dysbiosis. This study is necessary to assess the prospects for the creation of new metabiotics on the base of derivatives probiotics, that can effectively correct the altered microbial composition.

The objective of the research was investigation of the proliferative activity and biofilm formation by $E$. coli and $P$. aeruginosa in vitro under the influence of cell-free extracts of L. reuteri and B. bifidum.

\section{Materials and methods}

Commercial probiotic strains $B$. bifidum 1 (from medical product Bifidumbacterin-Biopharma, JSC Biopharma, Ukraine) and L. reuteri DSM 17938 (from medical product BioGaia, BioGaia AB, Sweden) were used as sources of biologically active structural components and as producers of metabolites. Cell free extracts were obtained by the authors' method (Knysh et al., 2018). Disintegrates were prepared by a multiple freezing-thawing of the suspension of probiotics with optical density of 10.0 units according to the McFarland scale (measured using the Densi-La-Meter device, Pliva-Lachema Diagnostika, Czech Republic) in physiological saline. Total of 10 cycles of freeze-thawing were carried out according to the following regimen: passive cooling (freezing) in freezing chamber of Samsung RB29FSRNDSA refrigerator down to $-23 \pm 1^{\circ} \mathrm{C}$, thawing in water bath at $37 \pm 1^{\circ} \mathrm{C}$ up to complete defrosting. Cultures of probiotics were obtained by cultivating of probiotic microorganisms in their disintegrates. To that end, the probiotic suspension in physiological saline of turbidity equaling 10.0 units on the McFarland scale was added into the disintegrate in 1:9 ratio and cultured at $37 \pm 1{ }^{\circ} \mathrm{C}$ for 72 hours in anaerobic conditions.

The disintegrates and probiotic cultures, grown in their disintegrates, were centrifuged at $1000 \mathrm{~g}$ for 30 minutes in order to remove remained cells and cellular debris. Supernatant was passed through sterile membrane filters with pore diameter of $0.2 \mu \mathrm{m}$ (Vladipor, Russia).

Five types of cell free extracts have been studied:

- L - filtrate of L. reuteri disintegrate;

- ML - filtrate of L. reuteri culture, grown in L. reuteri disintegrate;

- MLG - filtrate of $L$. reuteri culture, grown in L. reuteri disintegrate supplemented with $0.8 \mathrm{M}(73.7 \mathrm{~g} / \mathrm{L})$ glycerol and $0.4 \mathrm{M}$ (72.1 g/L) glucose;

- B - filtrate of B. bifidum disintegrate;

- MB - filtrate of B. bifidum culture, grown in B. bifidum disintegrate.

Clinical isolates: E. coli No. 22 and P. aeruginosa No. 23 (isolated from patients with gut dysbiosis and stored in the Collection of Microorganisms of the Laboratory of Prevention of Respiratory Infections of IMI NAMS, Kharkiv) were used as test cultures. The test cultures were cultivated overnight aerobically at $37^{\circ} \mathrm{C}$ on Mueller-Hinton agar (Merck, Germany).

Preparation of the inoculum: after verifying the purity of the culture, few colonies from the overnight incubated agar medium were emulsified in a physiological saline for obtaining a suspension of turbidity equaling 0.5 on the McFarland scale. The turbidity of the suspension was measured using the Densi-La-Meter.

Effect of cell-free extracts on proliferative activity of $E$. coli and $P$. aeruginosa was studied using modified Gladisheva's method (Gladisheva, 2014). Cell-free extracts and 10 times diluted inoculums were added into the test wells in the ratio of 9:1. Physiological saline and inoculums were added into the positive control wells $(\mathrm{PC})$ in the same ratio. The negative control wells (NC) contained only physiological saline. The plates were covered with lids and incubated aerobically for 1 hour at $35-37{ }^{\circ} \mathrm{C}$ in static conditions. After incubation, pepted meat broth (PMB; HiMedia, India) supplemented with $1 \%$ glucose aseptically was added into all wells of the microtiter plate in volume 2 times exceeding the total volume of cell-free extract and inoculum. The final concentration of microbial cells in the incubation medium was $\sim 10^{5} \mathrm{CFU} / \mathrm{mL}$. The optical density (OD) of the wells was measured at $578 \mathrm{~nm}$ using a microtiter-plate reader Lisa Scan EM (Erba Lachema s.r.o., Czech 
Republic) right after adding PMB and after incubation of plates aerobically for 24 hours at $35-37{ }^{\circ} \mathrm{C}$ in static conditions. The inhibition (or stimulation) index was calculated using the formula: $\mathrm{II}(\mathrm{SI})=(\Delta \mathrm{OD}-$ $\left.\Delta \mathrm{OD}_{\mathrm{PC}}\right) \div \Delta \mathrm{OD}_{\mathrm{PC}} \times 100 \%$, where $\Delta \mathrm{OD}$ and $\Delta \mathrm{OD}_{\mathrm{PC}}$ are the changes in optical density of the control and test samples within 24 hours.

Effect of cell-free extracts on biofilm formation by $E$. coli and $P$. aeruginosa. Biofilms were obtained in sterile 96-well polystyrene microtiter plates (JSC Eximcargotrade, Ukraine) by modified Stepanovic smicrotiter-plate technique (Stepanovic et al., 2007). The tryptone soya broth (TSB; HiMedia, India) supplemented with 1\% glucose aseptically was added into all wells of the microtiter plate. Cell-free derivative-containing extracts were added into the test wells to achieve a final concentration equaling $30 \%$ vol. Physiological saline was added to the positive control wells (PC) in the same volume. The inoculum was vortexed and then inoculated into a test and positive control wells of the microtiter plate. The final concentration of cells in the incubation medium was $\sim 10^{7} \mathrm{CFU} / \mathrm{mL}$. The negative control wells (NC) contained TSB supplemented with $1 \%$ glucose and physiological saline instead of cell-free extracts and inoculum. The plates were covered with lids and incubated aerobically for 24 hours at $35-37{ }^{\circ} \mathrm{C}$ in static conditions. After incubation, the contents of the wells were removed. Each well was washed three times with sterile $0.1 \mathrm{M}$ phosphate-buffered saline (PBS; $\mathrm{pH} 7.2$ ). The remaining attached biofilms were fixed by exposing them to hot air at $60{ }^{\circ} \mathrm{C}$ for $60 \mathrm{~min}$; they were stained by $1 \%$ crystal violet for $15 \mathrm{~min}$. After that, wells were washed ten times with distilled water. Ethanol was carefully added and then the microtiter plate was covered with the lid. It was left at room temperature for $30 \mathrm{~min}$. The optical density (OD) of the test and control wells was measured at $630 \mathrm{~nm}$ using a microtiter-plate reader Lisa Scan EM. Based on the OD values obtained for $\mathrm{NC}$ and test samples, biofilm formation was defined as: weak $\left(\mathrm{OD}_{\mathrm{NC}}<\mathrm{OD} \leq 2 \mathrm{xOD} \mathrm{NC}_{\mathrm{NC}}\right)$; moderate $\left(2 \mathrm{xOD}_{\mathrm{NC}}<\mathrm{OD} \leq 4 \mathrm{xOD}_{\mathrm{NC}}\right)$; strong $\left(4 \mathrm{xOD}_{\mathrm{NC}}<\mathrm{OD}\right)$; no biofilm formation $\left(\mathrm{OD} \leq \mathrm{OD}_{\mathrm{NC}}\right)$, according to the previously described method (In Leeet al., 2017).

The inhibition (or stimulation) index was calculated using the formula: II $(\mathrm{SI})=\left(\mathrm{OD}-\mathrm{OD}_{\mathrm{PC}}\right) \div \mathrm{ODPC} \times 100 \%$, where $\mathrm{OD}_{\mathrm{PC}}$ and $\mathrm{OD}$ are optical densities of the control and test samples, respectively.

All experiments were performed three times. Each sample assayed in triplicate. Average values of the obtained indices with standard deviations were determined $(\mathrm{x} \pm \mathrm{SD})$. One-way analysis of variance (ANOVA) followed by Bonferroni's post hoc multiple comparison test was performed. Differences were considered significant at $\mathrm{P}<0.05$.

\section{Results}

After the exposure of the test-cultures in the studied extracts for 1 hour at $35-37^{\circ} \mathrm{C}$ and the addition of pepted meat broth, the optical density of the test samples was significantly lower than of the control ones. This is evidenced by the data presented in the Table.

\section{Table}

The optical density of the test samples in relation to the controls after exposure of the test cultures in cell-free extracts $(\%, \mathrm{x} \pm \mathrm{SD}, \mathrm{n}=3)$

\begin{tabular}{lcccccc}
\hline \multirow{2}{*}{ Test cultures } & \multirow{2}{*}{$P C$} & \multicolumn{4}{c}{ Cell-free extracts } \\
\cline { 3 - 7 } & & $L$ & $M L$ & $M L G$ & $B$ & $M B$ \\
\hline E. coli & 100.0 & $36.5 \pm 1.8$ & $43.4 \pm 3.7$ & $44.3 \pm 5.3$ & $45.4 \pm 3.4$ & $40.2 \pm 2.2$ \\
P. aeruginosa & 100.0 & $37.5 \pm 3.7$ & $45.5 \pm 6.2$ & $46.7 \pm 5.9$ & $49.8 \pm 5.9$ & $46.0 \pm 7.2$ \\
\hline
\end{tabular}

Notes: PC - positive control; $\mathrm{L}$ - filtrate of $L$. reuteri disintegrate; $\mathrm{ML}$ - filtrate of $L$. reuteri culture, grown in $L$. reuteri disintegrate; MLG - filtrate of $L$. reuteri culture, grown in L. reuteri disintegrate supplemented with glycerol and glucose; $\mathrm{B}$ - filtrate of $B$. bifidum disintegrate; $\mathrm{MB}$ - filtrate of B. bifidum culture, grown in B. bifidum disintegrate.

As shown in Figure 1, the exposure of E. coli in cell-free extracts L, $\mathrm{B}$ and $\mathrm{MB}$ and the subsequent cultivation in the medium with $30 \%$ content of these extracts did not lead to significant changes in the optical density gain of the test culture compared to PC. Calculated for L, B and MB cell-free extracts SIs were $3.4 \%, 8.0 \%$ and $9.9 \%(\mathrm{P}>0.05)$, respectively. Exposure in the ML extract and subsequent cultivation of $E$. coli in the medium with its $30 \%$ content was accompanied by significant decrease in the optical density gain of the E. coli culture compared to
PC (II $=25.9 \%, \mathrm{P}<0.05)$. Exposure and cultivation of the test culture with MLG extract led to an even more significant decrease in the optical density gain of the E. coli culture compared to $\mathrm{PC}(\mathrm{II}=62.0 \%, \mathrm{P}<$ 0.005 ). The difference between the indicators of the optical density gain of the E. coli culture under the influence of the ML and MLG extracts was significant $(\mathrm{P}<0.05)$.

Thus, L, B and MB cell-free extracts had no significant effect on the proliferative activity of the $E$. coli in the conditions of this experiment. ML and MLG extracts had a significant inhibitory effect on the proliferative activity of $E$. coli. It is noteworthy that at the same concentration in the cultivation medium MLG extract had more pronounced inhibitory effect on the proliferation of the $E$. coli culture than ML extract.

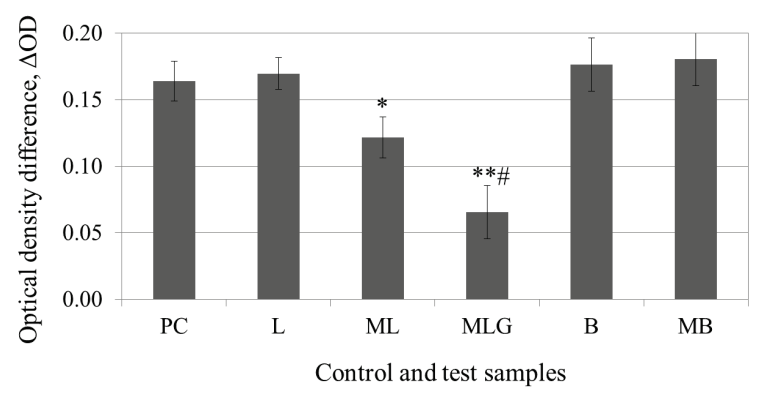

Fig. 1. The effect of cell-free extracts of $L$. reuteri and B. bifidum on the proliferation of $E$. coli (average $\triangle \mathrm{OD} \pm \mathrm{SD}$ at $578 \mathrm{~nm}, \mathrm{n}=3$ ): $\mathrm{PC}$ - positive control; $\mathrm{L}$ - filtrate of $L$. reuteri disintegrate; $\mathrm{ML}$ - filtrate of $L$. reuteri culture, grown in $L$. reuteri disintegrate; $M L G$ - filtrate of $L$. reuteri culture, grown in $L$. reuteri disintegrate supplemented with glycerol and glucose; $\mathrm{B}$ - filtrate of $B$. bifidum disintegrate; $\mathrm{MB}$ filtrate of B. bifidum culture, grown in B. bifidum disintegrate; the differences are significant compared to the $\mathrm{PC}:{ }^{*}-\mathrm{P}<0.05 ; * *-\mathrm{P}<$ 0.01 ; the differences are significant compared to the ML: ${ }^{\#}-\mathrm{P}<0.05$

The biofilm formation by the clinical isolate of $E$. coli was determined as strong. The data presented in Figure 2 suggest that the addition of all cell-free extracts to the cultivation medium leads to a significant decrease in the optical density of the eluates compared with eluate of PC. Thus, all studied extracts significantly inhibit biofilm formation by the clinical isolate of $E$. coli. In the presence of MLG and MB cell-free extracts in the cultivation medium the culture of $E$. coli still retains the ability for strong biofilm formation. II calculated for MLG extract was $22.6 \%(\mathrm{P}<0.05)$, for $\mathrm{MB}$ extract II equaled 26.8\% $(\mathrm{P}<0.05)$. The addition of $\mathrm{L}, \mathrm{ML}$ and $\mathrm{B}$ cell-free extracts was accompanied by the transition of the test-culture to the category with moderate biofilm formation. II calculated for L extract was $32.3 \%$ ( $\mathrm{P}<0.01)$, II for ML extract was $35.8 \%(\mathrm{P}<0.01)$ and for $\mathrm{B}$ extract II was $40.5 \%(\mathrm{P}<0.001)$.



Fig. 2. The effect of cell-free extracts derived from $L$. reuteri and B. bifidum on the biofilm formation by $E$. coli (average $\mathrm{OD} \pm \mathrm{SD}$ at $630 \mathrm{~nm}, \mathrm{n}=3$ ): $\mathrm{PC}$ - positive control; $\mathrm{L}$ - filtrate of $L$. reuteri disintegrate; $\mathrm{ML}$ - filtrate of $L$. reuteri culture, grown in $L$. reuteri disintegrate;

MLG - filtrate of $L$. reuteri culture, grown in $L$. reuteri disintegrate supplemented with glycerol and glucose; B - filtrate of B. bifidum disintegrate; $\mathrm{MB}$ - filtrate of $B$. bifidum culture, grown in B. bifidum disintegrate; the differences are significant compared to the PC: $*_{-} \mathrm{P}<0.05 ; * *-\mathrm{P}<0.01 ; * * *-\mathrm{P}<0.001$ 
As shown in Figure 3, exposure of $P$. aeruginosa culture in the L, B and $\mathrm{MB}$ cell-free extracts and its subsequent cultivation in a medium at $30 \%$ content of extracts caused no significant changes in the optical density gain of the test culture compared to PC. IIs calculated for L, B and $\mathrm{MB}$ cell-free extracts are $5.9 \%, 9.1 \%$ and $12.2 \%(\mathrm{P}>0.05)$, respectively. Exposure of the test culture in ML extract and subsequent cultivation in the presence of $30 \%$ concentration of this extract in the cultivation medium led to significant decrease in the optical density gain of the test-culture compared to PC (II $=53.0 \%, \mathrm{P}<0.001)$. It turned out that MLG extract even more inhibited the optical density gain of the testculture. II calculated for MLG extract was $96.9 \%(\mathrm{P}<0.001)$. The difference between the indicators of the optical density gain of the $P$. aeruginosa culture under the influence of the ML and MLG extracts was significant $(\mathrm{P}<0.01)$.

Obtained data allow us to conclude that L, B and MB cell-free extracts do not have a significant effect on the proliferative activity of the $P$. aeruginosa in the conditions of this experiment. ML and MLG extracts have a significant inhibitory effect on the proliferative activity of $P$. aeruginosa. It should be noted that MLG extract has more pronounced inhibitory effect on the proliferation of the $P$. aeruginosa culture than ML extract.

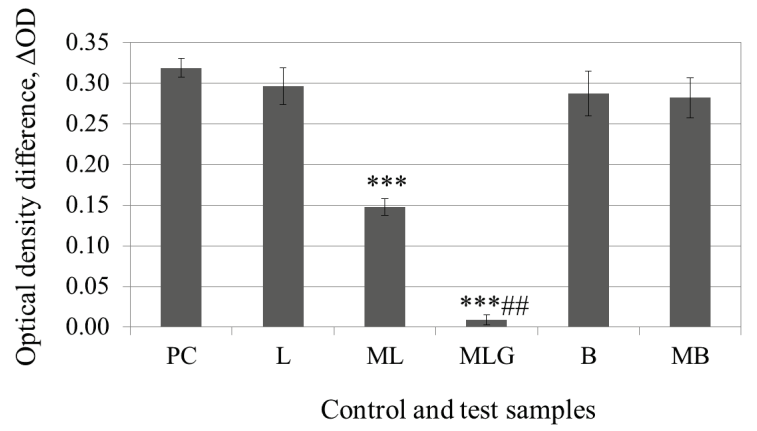

Fig. 3. The effect of cell-free extracts derived from $L$. reuteri and B. bifidum on the proliferation of $P$. aeruginosa (average $\triangle \mathrm{OD} \pm \mathrm{SD}$ at $578 \mathrm{~nm}, \mathrm{n}=3$ ): $\mathrm{PC}$ - positive control; $\mathrm{L}$ - filtrate of L. reuteri disintegrate; $\mathrm{ML}$ - filtrate of $L$. reuteri culture, grown in $L$. reuteri disintegrate;

$M L G$ - filtrate of $L$. reuteri culture, grown in $L$. reuteri disintegrate supplemented with glycerol and glucose; B - filtrate of B. bifidum disintegrate; $\mathrm{MB}$ - filtrate of $B$. bifidum culture, grown in B. bifidum disintegrate; the differences are significant compared to the $\mathrm{PC}: * * *-\mathrm{P}<$ 0.001 ; the differences are significant compared to the ML: ${ }^{\#}-\mathrm{P}<0.01$

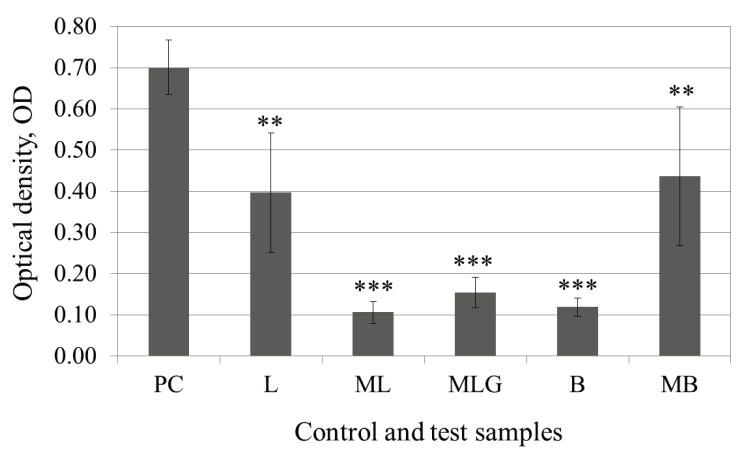

Fig. 4. The effect of cell-free extracts of $L$. reuteri and B. bifidum on the biofilm formation by $P$. aeruginosa (average $\mathrm{OD} \pm \mathrm{SD}$ at $630 \mathrm{~nm}$, $\mathrm{n}=3$ ): $\mathrm{PC}$ - positive control; $\mathrm{L}$ - filtrate of $L$. reuteri disintegrate; $\mathrm{ML}$ - filtrate of $L$. reuteri culture, grown in $L$. reuteri disintegrate; MLG - filtrate of $L$. reuteri culture, grown in $L$. reuteri disintegrate supplemented with glycerol and glucose; B - filtrate of B. bifidum disintegrate; $\mathrm{MB}$ - filtrate of $B$. bifidum culture, grown in B. bifidum disintegrate; the differences are significant compared to the PC:

$$
* *-\mathrm{P}<0.01 ; * * *-\mathrm{P}<0.001
$$

The biofilm formation by the clinical isolate of $P$. aeruginos $a$ was determined as strong. The data presented in Figure 4 suggest that the addition of all cell-free extracts to the cultivation medium leads to significant decrease in the optical density of the eluates compared to eluate of PC. The least pronounced decrease in the optical density of eluates was observed in the presence of $\mathrm{L}$ and $\mathrm{MB}$ extracts. IIs calculated for $\mathrm{L}$ and $\mathrm{MB}$ extracts equaled $38.2 \%(\mathrm{P}<0.01)$ and $32.3 \%(\mathrm{P}<0.01)$. Under the influence of $\mathrm{L}$ and $\mathrm{MB}$ extracts, the test-culture retained the ability of biofilm formation. Biofilm formation in the presence of these cell-free extracts was determined as strong. A more pronounced decrease in the optical density of eluates was observed under the influence of MLG extract. II calculated for MLG extract was $78.0 \%(\mathrm{P}<0.001)$. The biofilm formation by $P$. aeruginosa in the presence of MLG extract in the cultivation medium was determined as moderate. The most pronounced decrease in the optical density of eluates was observed under the influence of $\mathrm{ML}$ and $\mathrm{B}$ extracts. IIs calculated for $\mathrm{ML}$ and B extracts equaled $85.0 \%(\mathrm{P}<0.001)$ and $83.0 \%(\mathrm{P}<0.001)$. In the presence of $\mathrm{ML}$ and $\mathrm{B}$ extracts in the cultivation medium, the biofilm formation of the test-culture was determined as weak.

\section{Discussion}

The obtained data indicate that all the studied cell-free extracts have a more or less pronounced antimicrobial activity against the investigated gram-negative test cultures. A significant difference between the optical density of the test and control samples after hour exposure of the test cultures in cell-free extracts confirms it. The results of the study of the effect of cell-free extracts on the proliferative activity of the test cultures should be analyzed taking into account the peculiarities of the technique used. Fact is that after an hour of exposure of test-culture in undiluted test extract, the sample is diluted by nutrient broth. As a result of dilution, the concentration of the test substance was reduced to $30 \%$ vol. Test cultures that were exposed to the L, B and MB extracts after dilution completely restored the ability to proliferate over the following 24 hours, as evidenced by the same degree of optical density gain of the test and control samples. The proliferative activity of the test cultures that were exposed to the ML and MLG extracts was not completely restored after dilution over the following 24 hours. This is evidenced by a significantly lower in the optical density gain of the test samples compared to control ones. Thus, we can conclude that extracts containing the disintegration products of $B$. bifidum and $L$. reuteri, as well as extract containing metabolic products of $B$. bifidum, obtained when it was grown in its disintegrate, exhibit significantly less antimicrobial activity toward $E$. coli and $P$. aeruginosa than extracts containing metabolic products of $L$. reuteri, obtained when it was grown in its disintegrate without supplementation or supplemented with glycerol and glucose. Obviously, the similar effects of the studied extracts on the proliferative activity of $E$. coli and $P$. aeruginosa occured due to the same mechanisms of their action.

Antimicrobial activity of the metabolites of various lactic acid bacteria species was determined. Antimicrobial activities of $L$. acidophilus (Pyar et al., 2011; de Marco et al., 2018), L. casei subsp. pseudoplantarum, L. plantarum, and L. delbrueckii subsp. delbrueckii and different pools of Lactobacillus (Poppi et al., 2015), L. plantarum, L. fermentum and B. bidifum PTCC 1644 (Hesari et al., 2017), L. lactis, L. casei and L. reuteri (de Marco et al., 2018), L. plantarum, L. gasseri (Karimi et al., 2018) supernatants against $E$. coli was studied. Karimi et al. (2018) have detected the inhibitory effect of the supernatants of two probiotic lactobacilli (L. plantarum and $L$. gasseri) on the growth of four pathotypes of $E$. coli (enterotoxigenic, enteroaggregate, enteroinvasive and enteropathogenic) using two methods: disk diffusion and well diffusion agar methods. Poppi and co-authors reported that the supernatant from $L$. reuteti exhibited bactericidal activity toward pathogenic $E$. coli strain O157:H7. These researchers showed that $\mathrm{pH}$ adjustment (alkalinization) results in the decrease of antibacterial properties of the supernatants. The authors determined no direct relationship between the amount of lactic acid produced by lactobacilli and the degree of antimicrobial activity. This was a confirmation that the antimicrobial activity of lactobacilli is caused not only by the production of organicacids. Greifová et al. (2017) reported about high antibacterial potential of four L. reuteri strains against E. coli CCM 3988 and P. aeruginosa CCM 
3955 due to the production of organic acids, ethanol, and reuterin. In contrast to these results, other authors comparing of antimicrobial activity of supernatants of several probiotics (L. acidophilus, L. lactis, L. casei and $L$. reuteri, grown in absence or in presence of prebiotics) against $E$. coli have showed that the $L$. reuteri supernatant exhibits the least antimicrobial activity. Only the prebiotic combination improved the antimicrobial effect of $L$. reuteri supernatant on $E$. coli. However, it still remained lower compared to the activity of the supernatants of the other lactobacilli (de Marco et al., 2018).

Lactic acid bacteria has been reported to have antibacterial activity due to the ability to produce organic acids (lactic, acetic, propionic, butyric, succinic) and other antimicrobial compounds, such as hydrogen peroxide and bacteriocins, during growth (Sarkar \& Mandal, 2016; Jorgensen et al., 2017; Mokoena, 2017). Weak acids have higher antimicrobial activity than strong acids that are fully ionized in an aqueous solution (Poppi et al., 2015). It was demonstrated that only the protonated form of lactic acid has antimicrobial activity. Unlike the lactate anion, protonated form of lactic acid is membrane-permeant and enters the cells without using transporters or receptors. It acidifies the cytosol of most bacteria and provokes the cell death. The protonated lactic acid dominates at $\mathrm{pH} \leq 3.9$ (Tachedjian et al., 2017). In addition to the direct damaging effect on the cells, lactic acid can inhibit the growth of bacteria due to its chelating properties. It can capture elements necessary for growth (for example, iron) (Lebeer et al., 2008).

As shown by the results of the study, addition of glycerol and glucose to the disintegrate resulted in significant increase in the antimicrobial properties of the extract from $L$. reuteri culture grown in its disintegrate. It is known that some microorganisms can convert glycerin to reuterin. This non-protein water-soluble substance has antimicrobial, antifungal and antiprotozoan activity (Poppi et al., 2015; Spinler et al., 2017). Among all microorganisms, $L$. reuteti has the most pronounced ability to produce reuterin and has the least sensitivity to its antimicrobial action (Britton, 2017). We suppose that the most pronounced antimicrobial activity of the extract from the $L$. reuteri culture grown in its disintegrate supplemented with glycerol and glucose towards the test cultures occurs due to the content of reuterin in this extract. The obtained results confirm the promise of obtaining probiotic metabolites with bacteriotropic activity through precursor-directed biosynthesis.

The obtained data show that all studied extracts inhibit the biofilm formation by investigated test-cultures. The inhibition of biofilm formation by $E$. coli and P. aeruginosa under influence of probiotic derivatives was also achieved by other researchers. Therefore, some authors found out that the probiotic $L$. fermentum has the ability to attenuate the biofilm-forming potential of $P$. aeruginosa $\mathrm{PAO1}$ due to postbiotics: bacteriocins and exopolysaccharides (Sharma et al., 2018). Other authors have shown that lactic acid has an inhibitory effect on biofilm formation by P. aeruginosa through QS system (Kiymaci et al., 2018). De Marco et al. examined the ability of $E$. coli to form biofilm in the absence or presence of supernatants of several probiotic microorganisms: L. acidophilus, L. lactis, L. casei, L. reuteri and Saccharomyces boulardii. Supernatant of L. acidophilus, grown in de Man, Rogosa and Sharp (MRS) broth without supplementation or supplemented with inulin and isomaltose, was able to reduce the biofilm mass of $E$. coli. The addition of fructo-oligosaccharides exerted its anti-biofilm activity against $E$. coli. Metabolic products of $L$. casei, obtained in absence or in presence of prebiotics, showed an anti-biofilm activity against $E$. coli. Supernatants of $L$. lactis, $L$. reuteri and $S$. boulardii did not significantly reduce the biofilm formation by $E$. coli. It has been shown that the exopolysaccharides produced by $L$. acidophilus are able to inhibit the biofilm formation of gram-positive and gram-negative bacteria by affecting the expression of the genes involved in chemotaxis, auto-aggregation and co-aggregation (Miquel et al., 2016). In recent years special attention of researchers is focused on the biological role of biosurfactants. This group of various surface active molecules includes glycolipids, lipopeptides, polysaccharide-protein complexes, protein-like substances, lipopolysaccharides, phospholipids, fatty acids and neutral lipids. They are believed to perform a variety of physiological functions. Biosurfactants are involved in bacterial pathogenesis: adhesion and aggregation of cells, quorum sensing and biofilmformation. It has been shown that biosurfactants of lactobacilli have antimicrobial and antiadhesive properties (Gomaa, 2013).

It should be noted that all the authors, to whose works we refer, investigated the antimicrobial and anti-biofilm properties of supernatants obtained after growing lactobacilli in a traditional nutrient medium, MRS broth. In this study, we used filtrates of disintegrates and filtrates of cultures, grown in their disintegrates. That is, we used disintegrate as a cultivation medium for growing metabolite producers. This allowed us to obtain a metabolite-containing extract without admixture of the nutrient medium. In the process of disintegration by repeated cyclic freezing and thawing, part of the bacterial cells was destroyed. Both superficial and intracellular structural components entered the extracellular space. The surviving cells were exposed to thermal, osmotic, thermomechanical, dehydration and rehydration shock. Their metabolism was rearranged in response to stress. Bacterial cells were particularly sensitive to cold shock. The cold shock response is a cascade of cellular reactions, accompanied by a high level of temporarily expression of cold-induced proteins (CIPs). CIPs have the property to regulate multiple cellular processes, including proliferation and differentiation (Lindquist \& Mertens, 2018). Intracellular metabolites may leave cells exposed to temperature changes. Thus, bacterial disintegrate contains not only the structural components of cells (microbe-associated molecular patterns, MAMPs), but also bacterial cold shock proteins and other products of altered metabolism (damage-associated molecular patterns, DAMPs), which have powerful bioregulatory potential. During the cultivation of bacteria in disintegrates some of the components were used as nutrients. Actively growing and proliferating cells produce metabolic products into the extracellular space. Thus, the studied extracts were complex and different in composition. Probably the different components of the extracts may have different effects on biological processes in the cells. Cellular derivatives can act as signaling molecules and influence proliferation and biofilm formation by altering the expression of the genes involved in these processes. Supposedly, the disintegration products, which are structural components of lactic acid bacteria, can inhibit the biofilm formation by shielding cell surface adhesins and changing its physicochemical properties. Thorough study of biochemical composition of cell-free extracts would help clarifying the mechanisms of their bacteriotropic action and explain their effect on the proliferative activity and biofilm formation by test cultures.

\section{Conclusion}

The obtained results demonstrate that all cell-free extracts derived from $L$. reuteri and B. bifidum have more or less pronounced inhibitory effect on growth and biofilm formation by $E$. coli and $P$. aeruginosa. The discovered properties of cell-free extracts may contribute to their pharmaceutical applications. The extracts obtained from the culture of L. reuteri grown in its disintegrate without supplementation or supplemented with glycerol and glucose exerted the highest antimicrobial activity and can be used as metabiotics to prevent overgrowth of potentially pathogenic, as well as inoculation and proliferation of pathogenic gram-negative bacteria in the gastrointestinal tract. They can be used alone or in combination with cellular probiotics to enhance their probiotic action. This study also encourages further careful study of the biochemical composition of cell-free extracts and clarifying the mechanism of their action.

\section{References}

Bitto, N., \& Kaparakis-Liaskos, M. (2017). The therapeutic benefit of bacterial membrane vesicles. International Journal of Molecular Sciences, 18(6), 1287.

Britton, R. A. (2017). Lactobacillus reuteri. In: Floch, M., Ringel, Y., \& Walker, W. (Eds.). The microbiota in gastrointestinal pathophysiology: Implications for human health, prebiotics, probiotics, and dysbiosis. Academic Press, London. Pp. 89-97.

Carding, S., Verbeke, K., Vipond, D. T., Corfe, B. M., \& Owen, L. J. (2015). Dysbiosis of the gut microbiota in disease. Microbial Ecology in Health and Disease, 26(1), 26191

De Freitas, M. B., Moreira, E. A. M., Tomio, C., Moreno, Y. M. F., Daltoe, F. P., Barbosa, E., Neto, N. L., Buccigrossi, V., \& Guarino, A. (2018). Altered in- 
testinal microbiota composition, antibiotic therapy and intestinal inflammation in children and adolescents with cystic fibrosis. PLoS One, 13(6), e0198457.

Fuentes, S., \& de Vos, W. M. (2016). How to manipulate the microbiota: Fecal microbiota transplantation. In: Microbiota of the human body. Advances in experimental medicine and biology. Springer, Cham. Pp. 143-153.

Gladysheva, I. V. (2014). Antagonisticheskaia aktivnost korinebakterii [Antagonistic activity of corynebacteria]. Vestnik OGU, 174, 16-19 (in Russian).

Gomaa, E. Z. (2013). Antimicrobial and anti-adhesive properties of biosurfactant produced by lactobacilli isolates, biofilm formation and aggregation ability. The Journal of General and Applied Microbiology, 59(6), 425-436.

Gomes, T. A. T., Elias, W. P., Scaletsky, I. C. A., Guth, B. E. C., Rodrigues, J. F., Piazza, R. M. F., Ferreira, L. C. S., \& Martinez, M. B. (2016). Diarrheagenic Escherichia coli. Brazilian Journal of Microbiology, 47, 3-30.

Greifová, G., Májeková, H., Greif, G., Body, P., Greifová, M., \& Dubničková, M (2017). Analysis of antimicrobial and immunomodulatory substances produced by heterofermentative Lactobacillus reuteri. Folia Microbiologica, 62(6), $515-524$

Halmos, T., \& Suba, I. (2016). Physiological patterns of intestinal microbiota. (The role of dysbacteriosis in obesity, insulin resistance, diabetes and metabolic syndrome). Orvosi Hetilap, 157(1), 13-22.

Harmsen, H. J. M., \& de Goffau, M. C. (2016). The human gut microbiota. In: Schwiertz, A. (Ed.). Microbiota of the human body. Advances in experimental medicine and biology. Springer, Cham. 902, 95-108.

Hesari, M. R., Darsanaki, R. K., \& Salehzadeh, A. (2017). Antagonistic activity of probiotic bacteria isolated from traditional dairy products against $E$. coli O157: H7. Journal of Medical Bacteriology, 6(3-4), 23-30.

In Lee, S. H., Barancelli, G. V., de Camargo, T. M., Corassin, C. H., Rosim, R. E., da Cruz, A. G., Cappato, L. P., \& de Oliveira, C. A. F. (2017). Biofilm-producing ability of Listeria monocytogenes isolates from Brazilian cheese processing plants. Food Research International, 91, 88-91.

Jorgensen, M. R., Kragelund, C., Jensen, P. O., Keller, M. K., \& Twetman, S. (2017). Probiotic Lactobacillus reuteri has antifungal effects on oral Candida species in vitro. Journal of Oral Microbiology, 9(1), 1274582

Karimi, S., Rashidian, E., Birjandi, M., \& Mahmoodnia, L. (2018). Antagonistic effect of isolated probiotic bacteria from natural sources against intestinal Escherichia coli pathotypes. Electronic Physician, 10(3), 6534-6539.

Khodaii, Z., Ghaderian, S. M. H., \& Natanzi, M. M. (2017). Probiotic bacteria and their supernatants protect enterocyte cell lines from enteroinvasive Escherichia coli (EIEC) invasion. International Journal of Molecular and Cellular Medicine, 6(3), 183.

Kiymaci, M. E., Altanlar, N., Gumustas, M., Ozkan, S. A., \& Akin, A. (2018) Quorum sensing signals and related virulence inhibition of Pseudomonas aeruginosa by a potential probiotic strain's organic acid. Microbial Pathogenesis, 121, 190-197.

Knysh, O. V. (2019). Bifidogenic properties of cell-free extracts derived from probiotic strains of Bifidobacterium bifidum and Lactobacillus reuteri. Regulatory Mechanisms in Biosystems, 10(1), 124-128.

Knysh, O. V., Isajenko, O. J., Babych, J. M., Poljans'ka, V. P., Zachepylo, S. V., Kompanijec', A. M., \& Gorbach, T. V. (2018). Sposib oderzhannja biologichno aktyvnyh deryvativ bakterij probiotychnyh shtamiv [Method for obtaining biologically active derivatives of bacteria of probiotic strains]. Paten of Ukraine for useful model No 122859. Derzhavne Patentne Vidomstvo Ukrainy, Kyiv (in Ukrainian).

Lebeer, S., Vanderleyden, J., \& De Keersmaecker, S. C. J. (2008). Genes and molecules of lactobacilli supporting probiotic action. Microbiology and Molecular Biology Reviews, 72(4), 728-764.

Lin, L., \& Zhang, J. (2017). Role of intestinal microbiota and metabolites on gut homeostasis and human diseases. BMC Immunology, 18, 1.

Lindquist, J. A., \& Mertens, P. R. (2018). Cold shock proteins: from cellular mechanisms to pathophysiology and disease. Cell Communication and Signaling, 16,1 .
Litvak, Y., Byndloss, M. X., Tsolis, R. M., \& Bäumler, A. J. (2017). Dysbiotic Proteobacteria expansion: A microbial signature of epithelial dysfunction. Current Opinion in Microbiology, 39, 1-6.

Martinez de la Peña, C. F., Armstrong, G. D., Arenas-Hernández, M. M. P., \& Cieza, R. J. (2016). Homeostasis vs. dysbiosis: Role of commensal Escherichia coli in disease. In: Torres, A. G. (Ed.). Escherichia coli in the Americas. Springer, Cham. Pp. 281-299.

Matamouros, S., Hayden, H. S., Hager, K. R., Brittnacher, M. J., Lachance, K. Weiss, E. J., Pope, C. E., Imhaus, A.-F., McNally, C. P., Borenstein, E., Hoffman, L. R., \& Miller, S. I. (2018). Adaptation of commensal proliferating Escherichia coli to the intestinal tract of young children with cystic fibrosis. Proceedings of the National Academy of Sciences, 115(7), 1605-1610.

McCarville, J. L., Caminero, A., \& Verdu, E. F. (2016). Novel perspectives on therapeutic modulation of the gut microbiota. Therapeutic Advances in Gastroenterology, 9(4), 580-593.

Miquel, S., Lagrafeuille, R., Souweine, B., \& Forestier, C. (2016). Anti-biofilm activity as a health issue. Frontiers in Microbiology, 7, 592

Mokoena, M. P. (2017). Lactic acid bacteria and their bacteriocins: Classification, biosynthesis and applications against uropathogens: A mini-review. Molecules, 22(8), 1255.

Peh, K. K., Pyar, H., \& Liong, M.-T. (2011). Inhibitory effect of metabolites from probiotics Lactobacillus acidophilus strains on growth of pathogenic bacteria Journal of Pharmacology and Toxicology, 6(5), 533-540.

Sarkar, A., \& Mandal, S. (2016). Bifidobacteria - insight into clinical outcomes and mechanisms of its probiotic action. Microbiological Research, 192, 159-171.

Scales, B. S., Dickson, R. P., \& Huffnagle, G. B. (2016). A tale of two sites: How inflammation can reshape the microbiomes of the gut and lungs. Journal of Leukocyte Biology, 100(5), 943-950.

Sharma, V., Harjai, K., \& Shukla, G. (2017). Effect of bacteriocin and exopolysaccharides isolated from probiotic on $P$. aeruginosa PAO1 biofilm. Folia Microbiologica, 63(2), 181-190.

Shenderov, B. A. (2013). Metabiotics: Novel idea or natural development of probiotic conception. Microbial Ecology in Health and Disease, 24(1), 20399.

Singh, A., Vishwakarma, V., \& Singhal, B. (2018). Metabiotics: The functional metabolic signatures of probiotics: Current state-of-art and future research priorities. Metabiotics: Probiotics effector molecules. Advances in Bioscience and Biotechnology, 9(4), 147-189.

Spinler, J. K., Auchtung, J., Brown, A., Boonma, P., Oezguen, N., Ross, C. L., Luna, R. A., Runge, J., Versalovic, J., Peniche, A., Dann, S. M., Britton, R. A., Haaga, A., \& Savidge, T. C. (2017). Next-generation probiotics targeting Clostridium difficile through precursor-directed antimicrobial biosynthesis. Infection and Immunity, 85, 10.

Stecher, B. (2015). The roles of inflammation, nutrient availability and the commensal microbiota in enteric pathogen infection. Microbiology Spectrum, 3, 3 .

Stepanović, S., Vuković, D., Hola, V., Bonaventura, G. D., Djukić, S., Ćirković, I., \& Ruzicka, F. (2007). Quantification of biofilm in microtiter plates: Overview of testing conditions and practical recommendations for assessment of biofilm production by staphylococci. APMIS, 115(8), 891-899.

Tachedjian, G., Aldunate, M. Bradshaw, C. S., \& Cone, R. A (2017). The role of lactic acid production by probiotic Lactobacillus species in vaginal health. Research in Microbiology, 168(9-10), 782-792.

Valdes, A. M., Walter, J., Segal, E., \& Spector, T. D. (2018). Role of the gut microbiota in nutrition and health. British Medical Journal, 361, k2179.

Winter, S. E., \& Bäumler, A. J. (2014). Dysbiosis in the inflamed intestine: Chance favors the prepared microbe. Gut Microbes, 5(1), 71-73.

Yu, L. C. H. (2018). Microbiota dysbiosis and barrier dysfunction in inflammatory bowel disease and colorectal cancers: Exploring a common ground hypothesis. Journal of Biomedical Science, 25(1), 79. 